\title{
Indicadores bioculturales en proyectos de gestión ambiental. 日 caso de la meliponicultura en Yucatán
}

\author{
Mauric io López Barreto \\ Cephcis-UNAM, Mérida, México \\ Email: mauri.lopez@gmail.com \\ Miguel Pinkus Rendón \\ Cephcis-UNAM, Mérida, México \\ Email: tmapinkus@humanidades.unam.mx
}

Recibido: 14.06 .20 | Aceptado: 13.08 .20

\begin{abstract}
Resumen: El presente trabajo revela diferentes criterios y metodologías para el ejercicio de evaluación de proyectos de gestión ambiental. Aunado a la carencia de instrumentos de evaluación que incorporen indica dores de impacto de la dimensión biocultural, se constata la tendencia a privilegiar una valoración mercantilista de proyectos, incidiendo en la gestión ambiental. Con el apoyo teórico de la valoración relacional (relational values) y a tra vés de la categoriza ción de códigosemergentes de datos recabadosa través de métodos etnográfic os, el presente trabajo concluye con una propuesta para la a plic a ción de indic a dores para conocerla incidencia en la dimensión biocultural en el caso de proyectospara el rescate de la meliponic ultura, es decir, el cultivo de la abeja nativa comúnmente conocida como abeja melipona o Xunancab (en Maya) (Melipona beecheii), en la península de Yucatán.

Palabras claves: Gestión ambiental; maya peninsular, evaluación; indicadores.
\end{abstract}

\section{Bioc ultural indic ators in environmental management projects. The case of meliponiculture in Yucatan}

\begin{abstract}
The present work reveals different criteria and methodologies for the exercise of the evaluation of environmental management projects. Together with the lack of evalua tion instruments that incorporate impact indicators of the biocultural dimension, the tendency to privilege a mercantilist valuation of projects, with emphasis on environmental management, is confimed. With the theoretical support of relational values and through the categorization of emerging codesfrom data collected through ethnographic methods, this work concludes with a proposal for the application of indicators to know the impact on the biocultural dimension in the case of projects to rescue meliponiculture, that is, the cultivation of the native bee commonly known as melipona bee or Xunancab (in Maya) (Melipona beecheii), in the Yucatan peninsula.
\end{abstract}

Keywords: Environmental management; peninsular Maya; evaluation; ind ic ators.

\section{Indic adores bioc ulturais em projetos de gestão ambiental. O caso da meliponicultura em Yucatán}

Resumo: O presente trabalho revela diferentes critérios e metodologias para o exercício de a valiação de projetos de gestão ambiental. Adicionado à carência de instrumentos de 
a valiação que incorpora indicadores de impacto da dimensão biocultural, se constata a tendência de privilegiar uma avaliação mercantilista de projetos, incidindo na gestão a mbiental. Com o apoio teórico da valoração relacional (relational values), e por meio da categ orização de códigosemergentes de da dosrecuperadospormétodosetnográfic os, o presente traba lho conclui com uma proposta para a plic a ção de indic a dorespara conhecer a inc idência da dimensão biocultural no c aso de projetospara o resga te da meliponic ultura, quer dizer, o cultivo da abelha nativa comumente conhecida como abelha melipona ou Xunancab (em Maya) (Melipona beecheii), na península de Yucatán.

Palavras-chave: Gestão a mbiental; maya peninsular; avaliação; indicadores.

Como citar este artículo: López Ba rreto, M. y Pinkus Rendón,M. (2020). Indicadores bioc ultura lesen proyec tos de gestión a mbiental. El ca so de la meliponic ultura en Yucatán. Polis Revista La tino a meric a na, (57), 52-72. doi: http:// dx.doi.org/10.32735/S0718-6568/2021N57-1564

\section{Introducción}

Los pueblos orig ina rios históric a mente han protegido a lrededor del $80 \%$ de la biodiversidad del planeta (Rundle, 2019) a tra vés de la apropiación del temitorio y de la naturaleza desde una lógica fundamentada en la gestión de la diversidad biocultural de cada grupo (Maffi, 2014; UNESC O, 2008). Por otro la do, iniciativas públicas para la conservación de la biodiversidad diseñadas bajo la óptica neoliberal, de mercado y crecimiento económico, han conducido, paradójicamente, a la pérdida de la biodiversidad (Otero et al., 2020).

Según Durand (2014) el esquema de conservación de la biodiversidad en México está enmarcado en una lógica neoliberal, que ha generado resultados ambiguos, frecuentemente en detrimento de la relac ión de lascomunidadescon su ento mo natural. Este esquema promueve procesos como la comodificación (derivado de commodities), es decir, la asignación de un valor monetario a algunos elementos de la naturaleza u otras cosas o fenómenos que no eran objeto de intercambio comercial, así como la adopción de políticas públic as para incentivar la privatización y la ampliación de los mercados.

Tal esquema neoliberal, ajeno a la cultura local de los pueblos oniginarios, ha generado importantes dislocaciones en el modo de producción tradicional de estas comunidades, alterando el uso del suelo y del a gua, destruyendo recursosnatura les y reduciend o a monocultivos los sistemas diversificados de produc ción múltiple característicos del patrimonio bioc ultural de los pueblos originarios y convirtiendo las familias campesinas en «unidades de producción especia liza das y dependientes» (Durand, Figueroa, Guzmán, 2011).

En este contexto, los primeros esfuerzos para integrar las diferentes dimensiones de la sustentabilidad-como la cultura y la consenvación-en la evaluación de proyectos productivos utiliza ron casi exclusiva mente a nálisis monetarios, en particular de costo-beneficio. Estas propuestas, han demostra do serias limita ciones para su a plica ción como criterio únic o en la evalua ción de la sustentabilidad de proyec tos para el ma nejo de recursos natu- 
ra les, en particula r en contextos campesinos de países en vía s de desa rro llo (Martínez-Alier, Munda y O'Neill, 1998).

En México, por ejemplo, el Consejo Nacional de Evaluación de la Política de Desarrollo Social (CONEVAL) «tiene por objeto nomar y coordinar la evaluación de las políticas y programas de desarrollo social que ejecuten las dependencias públicas, y establecer los lineamientos y criterios para la definic ión, identificación y medición de la pobreza», fundamenta do en la Ley General de Desa rollo Social (LGDS) (CONEVAL, s.f.). Es de señalar que esta medición enfatiza la dimensión monetaria como medida central para la evaluación de los programas soc io-productivos (Ochoa, 2007).

Para J oseph Stiglitz, las métricas de evaluación inadecuadas han lleva do a políticas ineficientes, señala que si los indic adores solo se concentran en el bienestar material (por ejemplo, en la producción de bienes, más que en la salud, la educación y el ambiente), la sociedad se vuelve más materialista, resaltando, «si medimos lo incorrecto, ha remos lo incorrecto» (Stiglitz, 2019). Se puede decir, que el objetivo de un indicador es controlar la evolución de una parte de la realidad.

La Organización para la Cooperación y el Desa rollo Económico (OCDE), se refiere a un indicador como un parámetro o valor derivado de parámetros que proporciona información para describir el esta do de un fenómeno, a mbiente o área, con un significa do que va más allá del direc tamente a socia do con el valor del parámetro mismo (OECD, 2003). Aunque en algunas fuentes parámetro e índice se utilizan indistintamente como sinónimos de indicador, no tienen el mismo significado. El primero se refiere a una propiedad medible u observa ble, mientrasque el índ ice esun a grega do de pará metroso ind ic a dores (RodríguezOrtega y Flores-Martínez, 2008).

En este sentido, y en concordancia con García-Ba mios y García-Ba mios (2008), el trabajo realizado propone un sistema de evaluación que ofrezca a los tomadores de decisiones o a los partic ipantes en el proyecto, los siguientes instrumentos:

1. una «ventana abierta»que permita observar el funcionamiento del proyecto;

2. un sistema que permita interpretar, en forma consistente, su funcionamiento, y resultadosy

3. un sistema de medic ión que permita, controlarlosprocesosy decidirca mbioscuando fuere necesario, ya sea al inic io o durante la ejecución y operación del proyecto.

Por lo tanto, la utilidad del sistema de indicadoresyace en que cada uno de ellos debe ser un instrumento de observación, de interpretación o de control, según corresponda, de tal manera que pueda servir al tomador de decisiones para conduc ir el proyec to a su propósito final. De esta manera, cada indicador, además de representar un componente físico o relacional medible susceptible de ser modifica do, debe ser considerado como un componente que está subordinado y es guiado por un propósito final que opera a partir de valo- 
res, creenciasy expectativas del toma dorde dec isiones(Will, 1988, en García-Ba miosy Ga rcíaBa mios 2008). Esasí, que el presente tra bajo reconoce que la ela boración del instrumento de evaluación no admite una construcción neutra, por el contrario, el instrumento refleja un sesgo del investigador sobre cómo debe orientarse el proyec to, a partir de la interpretación de los datos generados por el mismo.

El concepto de diversidad biocultural ha cobrado mayor relevancia en las últimas décadas, ya que se perfila como una a ltema tiva holística al modelo prevaleciente, destacando la relación inalienable y simbiótica entre la cultura de los pueblos locales y originarios y la biodiversidad (Bridgewater y Rotherha m, 2019; Maffi; 2014). Maffi (2014, p. 7) señala que «a diversidad biocultural es la 'verdadera' red de vida (web of life), definida como la diversidad de la vida entrelazada en la naturaleza y la cultura, un todo integrado y formado por la biodiversidad, diversida d c ultural y diversidad lingüístic a». La opera ciona liza ción del c oncepto, a través de indicadores, entonces, busca revalorizar y promover esta dimensión dentro del proyecto.

Es importante hacer hincapié en que lo biocultural representa la diversidad ontológica común de los pueblos originarios. Para Arturo Escobar, entre otros a utores, esta diversidad se manifiesta en los movimientos sociales, anclados en un entendimiento profundo de la vida, donde se interrelacionan los componentes - materiales e inma teriales; huma nosy nohumanos-como saberes, práctic asy creencias; funda menta dosen una ontología relacional dentro de una delimitación temitorial, es decir, de espacios-tiempos vitales para las comunida des (Esc obar, 2015). En este sentido, las comunida des indígenas no son solo cuidadoras y reproductoras de comunes, sino creadoras del mismo, a tra vés de demandas y reivindicacionesfrente a un proyec to único hegemónic o homogeneizadorneoliberal, en defensa de la diversidad ontológica.

En Yucatán, la meliponicultura, o el manejo de las abejas nativas Xunancab (en Maya) (Melipona beecheii), esuna práctic a milena ria que, a pesarde su evidente a raigo biocultural en la región, fue ampliamente desatendida y en muchos casos sustituida por la a pic ultura (Apis mellifera), por su mayor rentabilidad comercial (Rodríguez-Balam y Pinkus-Rendón, 2015). Adicionalmente, la meliponicultura ha confrontado diversas amenazas, como el cambio climático, el cambio de uso del suelo, y la pérdida de saberes y prácticas asociadascon esta actividad (Quezada-Euán, 2018).

No obstante, a utores sostienen que en los últimos a ños se ha incrementa do el número de grupos de meliponicultores en la Península de Yucatán (Hirst, 2018; Pearson, 2014; Main, 2012).

Ca be seña larque una enc uesta rea liza da entre el año 2000-2005 en el esta do de Yuca tánentidad con mayor concentración de meliponic ultura tra dicional de la Península-reveló que el $65 \%$ de los meliponicultores tienen una edad media de 56 años y que $22 \%$ de meliponic ultores son mujeres (G onzá lez y Quezada-Euán, 2010). Estos da tos revelan un pa- 
pel signific a tivo de adultos ma yores y de mujeres en la revaloración de esta práctica tra dicional y sus componentes.

Por lo tanto, es necesario desarrollar un sistema de indicadores que permita la incorporación de componentes de la dimensión biocultural. En este sentido, el presente trabajo se a poya en la Teoría de losValoresRelacionales, para elaboraruna propuesta de indicadores que describen la incidencia de losproyec tosde meliponic ultura en el patrimonio biocultural local, y contribuir a su revaloración, fomento y conservación.

\section{E manejo de la biodiversidad en comunidades mayas frente al modelo neoliberal}

La riqueza de losconocimientos localessobre la flora y fauna en lascomunidadesmayasse refleja en la adopción de una estrategia de uso múltiple de los sistemas socio-ecológicos (SSE) que tiene, al menos, seis componentes: la milpa donde se cultiva el maíz y otras especies, el huerto familiar que tienen en promedio entre 50 y 100 especies; las selvas sec undarias, las selvas ma duras, las selvas maneja das y loscuerpos de a gua; y en práctic ascomo la meliponic ultura, donde destacan sus detallados conoc imientos sobre las abejas nativas, a sí c omo la caza y la pesca. Para Toledo y Ba rrera-Bassols (2008), esta capacidad de adaptación a las condiciones locales y especialmente el aprovechamiento de la biodiversidad local y regional por parte de las poblaciones humanas constituye un ejemplo de losatributos positivos del patrimonio biocultural.

Debid o a su c lima, en Yuc atán existen entre 2.400 y 3.000 espec ies de plantas, «de lascua les unas 2.200 o 2.400 se restringen a la porción mexicana. Esta diversidad florística se ve reflejada en el detallado conocimiento maya de las plantass (Toledo y Barrera-Bassols, 2008, p. 152). Según losautores, Flores (en un estudio de 2001) «reportó nombres loca les para $88 \%$ de las 260 especiesde leguminosas, que es la fa milia mejor representada en la península »(lbid). Por ejemplo, los solares, «se loca lizan a Irededorde lascasas y tienen una superfic ie de entre 500 y 2.000 metros cua dra dos, con máximos de hasta 5.000» (Toled o y Ba rrera-Bassols, 2008, p. 153); a llí «se cultivan, toleran y manejan una gran cantidad de especiesde plantas, principalmente árboles y a rbustos, a demás de a nima les doméstic os como cerdos, gallinas, guajolotes, patos y colonias de abejas, que son fundamentales en la alimentación familiar»(lbid).

En estos lugares es donde se pueden localizar abejas meliponas, ya que desde tiempos ancestrales se aprovechaba la miel de la abeja Xunancab, alimentadas de plantas del solar y utilizada para ceremonias o primicias, como el hanlikol-que aún se realizan en el solar-para invocar el buen tiempo y las buenas floraciones de las plantas. Asimismo, especies como el ciruelo (Spondias mombin) suelen a coger en sus oquedades a los nidos de las abejas kantsac (Scaptotringona pectoralis), las mejenbol (Nannotringona perilampoides), las uska'ab (Plebeia spp.) y las minúsc ulas pu'up (Trigonisca maya) (González y De Araujo, 2014, p. 243-251). 
En este sentido, se puede consta tar que el uso-múltiple de los SSE, en particular, la milpa, el huerto y el monte, provee una abundancia de especies de flora que promueve, a su vez, la abundancia de especies de abejas, favoreciendo la másimportante función ecológica de las abejas como es la polinización.

Por otro lado, la capacidad de las comunidades de reproducir, transmitir y promover su patrimonio biocultural de manera colectiva proporciona un sentido de identidad para un proyecto de vida, suministrando los elementos necesarios para reconstruir la memoria de eventosocumidos en el pasado, y para la planeación a futuro con el objetivo de mitigar el impacto de sucesos inesperados, por ejemplo, las consecuencias adversas del cambio climático (Toledo y Barrera-Bassols, 2008, p. 205).

En los huertos mayas-peninsula res, se esta blec en la s relac iones socia les entre los parientesy los miembros de la comunidad que se ven fortalecidas a través de vínculos de confianza y de reciprocidad. Estas relaciones benefician a una red de familia y vecinos, quienes entre todos se regalan, intercambian o comercializan los productosa pequeña escala. Además, el solar es un lugar de educación familiar «que permite la creación de identidad y esparcimiento», y una herencia clave para el futuro de los descendientes, quienespotenc ia Imente se beneficiaran de los bienes, los conocimientos y de las experiencias compartidas en estos espacios (Cahuich, 2010, en Chá vez, 2014, p. 271).

A pesar de la prevalencia de usosy costumbrestra diciona lesen estas comunida des existen serias amenazas a este modo de vida tales como la migración, pérdida del idioma, cambio de culto religioso, etc. (Ramírez, 2006). En el contexto de la conservación de la biodiversidad, políticas públicas enmarcadas en el modelo neoliberal han intentado promover actividades productivas con fines puramente mercantiles. Se ha considerado, por ejemplo, que la instrumentación de proyectos de ecoturismo en áreas protegidas se convierta en negocios renta bles para las comunida des y podrán compensa r los costos de conservación/destrucción de los recursos naturales, a lo cual se agrega la reducción en la disponibilidad y el acceso a los recursos, lo que hipotéticamente promovería el interés local por la consenación (CONANP, s.f.). Para Sarukhán (2009), de esta manera se busca impulsar a los habitantes rurales a tranformar los paisajes y otros elementos de su entomo y cultura en nuevas mercancías, ponerles un precio monetario, y transformar a ello mismos, en empresarios y a dministra dores del capital na tural.

Para contra rrestar esto, algunos autores han propuesto incentivar el rescate de la meliponic ultura, a través de un abordaje técnico-productivista, con fines comerciales de los produc tos deriva dos de la abeja nativa Xunancab, tal como los productos medic inales, entre otros (González, Quezada-Euan, y Medina-Medina, 2006). No obstante, el presente trabajo propone contribuir a la desmercantilización de la meliponicultura y a su revalorización desde un enfoque relac ional, funda menta do en el pa trimonio biocultural ma ya, como altemativa al modelo mercantilista, de corte neoliberal. 


\section{La evaluación de Sistemas Socio-Ecológicos (SSE) y la dimensión biocultural}

Desde los años 90 los diferentes sistemas de evalua c ión de proyectos de gestión a mbiental en los que participan comunidades locales y pueblos originarios se caracterizaron por un sesgo a gríc ola-ambiental. Otros sistemas tratan de incluir, a tra vés de indicadores, las presiones que ejercen las actividades humanas sobre el a mbiente al induc ir cambios en el estado de los ecosistemas (Galván-Miyoshi, Masera y López-Ridaura, 2008, p. 45). Con la expansión del modelo neoliberal, los economistas desa rollaron y refinaron métodos de evaluación intentando incorporar el valor de los servicios ecosistémicos y poder integrar éstos al libre mercado. Para esto hubo que asigna rles un valor monetario tra nformando el valor de los bienes y servicios de la naturaleza, en capital natural (Durand, 2014, p. 190).

Los sistema s c onvenc iona les de eva lua c ión para proyec tos de gestión a mb iental en los SSE, derivados de un esquema neoliberal, basados en una perspectiva de valor, genera Imente de tipo monetario, han privilegiado solo algunos de sus atributos, a costa de la pérdida de otras dimensiones inmanentes a la naturaleza del proyecto. Resulta por tanto necesario, incorporar en las metodologías de evaluación otros componentes que representen las múltiples dimensiones que integran los SSE. Un primer paso en esta dirección será el de identific ar a quellas dimensiones no considera das en las metodologías convencionales. En los últimos años se han desarrolla do diversas propuestas metodológicas para la integración de nuevos indica dores de SSE (Galván-Miyoshi, 2008).

Recientemente han surgido inic ia tivas para integrar ind ic a dores funda menta dos en la Teońa de los Valores Relac ionales (Chan et al., 2016; Schröter et al., 2020; Kleespies y Dierkes, 2020), a sí c omo, en los va lores de la diversidad biocultural (DeRoy, Darimont y Service, 2019; Dac ks, Tic ktin, Mawyer, et al., 2019). Los va lores rela c ionales serían los va lores desea bles de las relacionesentre las personasy la natura leza y entre personas (a tra vés de la na turaleza) (IPBES, 2018). Para Chan et al. (2016, p. 1462), la teoría de la valoración rela cional refleja el compromiso y la relación que los seres humanostienen con la naturaleza y con su hábitat. La noción relacional en los valores prevalece en una amplia parte de la humanidad, incluyendo entre la comiente de pensamiento de los clásicos (ej. aristotélicos), el occidental contemporáneo, el indígena (ej. tsawalk, sumak kawsay), el feminista (ej. ética del cuidado), y filosofía s del este (ej. confuciana, budista). La noción de una vida plena, fundamentada en las relaciones, se expresa en diversas cosmovisiones, incluyendo en Ubuntu de África del Sur, en la ec onomía Gandhia na en la India, en el Buen Vivir en va rios pa íses de América Latina y en movimientos norteamericanos «de vuelta a la tierra» (back to the land). Asimismo, los cinco «fundamentos morales» más comunes entre muchas personas-pureza/santidad, a utoridad/respeto, grupo/lealtad, justicia/reciprocidad y daño/ cuidado-son mejorcomprendidos desde la óptica de relacionesy una vida plena, que a través de los valores instrumentales (fundamentales en la economía neoclásica y ambiental) o valores intrínsecos (fundamentales en la economía ecológica) (Chan et al., 2016, p. 1463). 
Para muchos su identidad cultural y bienestar se derivan de las relaciones entre humanos, flora y fauna, u otros objetos; en un lugar particular. El cuidado y mantenimiento de estos luga respueden seresencialespara prácticascultura lesy losva loresc entra les, por medio de una visión colectiva de temitorio (Chan et al., 2016, p. 1464). De esta manera, la teoría de la valoración relacional involucra tanto el colectivo humano, como a los individuos. Los objetosnatura lesno tienen valores relac iona lesperse, pero losva lores rela ciona les resultan de la relación o compromiso hacia ellos. La teońa de la valora ción rela cional no es un fin en sí, pero permite visua liza rimporta ntes fac tores para la conserva ción, sustenta bilida d y la compatibilidad de vivir una vida plena en equilibrio con la naturaleza.

En la península de Yucatán, por ejemplo, la conexión entre los valores relacionales y la conservación de la biodiversidad se puede evidenciar en los movimientos que integran comunida desy orga niza ciones loca lesformando redes de a poyo para la reivindic ación de modelos agroecológic os sustentables y por la defensa del temitorio y del patrimonio cultural, en rechazo a esquemasa groind ustria les y todos susderiva dos (ej. la ta la de la selva, uso insustentable delagua para riego de monocultivos, destrucción del hábitat de poliniza dores, entre otros) (López, 2015).

En este sentido, cuando las polític as se enfoc a n en va lores instrumentales y tomas de decisionesorienta das por la economía, losesfuerzos de conserva ción son pueden serafectados de manera adversa. El impacto negativo a la biodiversidad bajo este enfoque se puede evidenciar en los efec tos a dversos de los monoc ultivos a groind ustriales, entre otras prácticas característic as de una lógica ec onomic ista (Allen, Quinn, English y Quinn, 2018).

Por lo tanto, la teońa de la valoración relacional puede ser una herramienta de gran utilidad para lograrun uso más sustentable de la naturaleza. Pa ra algunos a utores los valores rela c iona les se reflejan en va lores como la conexión con la naturaleza, la comunalidad, la identidad, el parentesco, y el cuida do del temitorio (Kleespies y Dierkes, 2020; Sc hröter et al., 2020).

En el mismo sentido, con la finalidad de elaborar la primera propuesta pa ra medir la riqueza biocultural a escala global, en G lobal Index of Biocultural Diversity, Loh y Hamon (2005) determinaron en una ecuación el concepto de diversidad biocultural, utilizando el número de idiomas, religiones, y grupos étnic os presentes dentro de cada país como variables de diversida d cultural; a sí c omo, el número de especies de pája rosy ma míferos, y el número de especies de plantas como medida de su diversidad biológica.

Por tanto, el índice de diversidad biocultural desa rollado está compuesto por tres partes:

1. Un componente de la riqueza de diversidad biocultural, que es una medida relativa de la diversidad biocultural en su totalidad, es decir, sin a justar el conteo total de cada uno de loscinco indicadores.

2. Un componente de superfic ie, que ajusta los indicadores a la superfic ie terrestre, y, 
portanto, mide la extensión física de la diversidad cultural relativa de un país.

3. Un componente poblacional, que ajusta los indicadores a la población humana y, por tanto, mide la diversidad biocultural con relación al tamaño poblacional de un país.

Cabe señalarque, debido a su plantea miento global (macro), el índice ca rece de elementos relacionadoscon la gestión a mbiental local, como el uso y manejo de las especies, y la apropiación de nuevas tec nologías para actividades productivas, por ejemplo.

Un artíc ulo de reciente public ación, sobre indic adores biocultura les en a poyo a la gestión y el monitoreo a mbiental rea liza do por comunida des locales (DeRoy et al., 2019), proponen criterios generales, para guiar la selección de indica dores biocultura les como altemativa a un enfoque ecológico funcional. Los autores plantean que los indicadores deben ser: cultura Imente apropiados, inclusivos, sensibles a los impactos, que apoyan las relaciones basadas en un sentido de lugar, perceptibles, y vincula dos al bienestar humano.

En este sentido, cabe destacar que la relevancia y el potencial de resurgimiento de la gobemanza de los pueblos originarios es prueba fehaciente que el enfoque biocultural, a tra vés de la gestión de su entomo a mbiental ha promovido la resiliencia socio-ecológica, el bienestar humano y la integridad ecosistémic a durante miles de años, así como las metas de objetivos de sustenta bilidad g lobales (DeRoy et al., 2019). Por lo ta nto, es a tra vés del enfoque biocultural que el presente estudio busca incorporar estos elementos en el instrumento de evaluación.

Dacks et al. (2019) discuten acerca de integrar las siguientes dimensiones de factores soc ioc ultura les rela ciona das con la diversidad biocultural: conectividad con las personasy lugar, y conoc imientos, habilidades, prácticas, cosmovisiones y valores indíg ena s loc ales. El estudio también destaca la existencia de pocas iniciativas para desa rollar indicadores de percepción, cuya omisión resulta en una carencia importante del aspecto cultural del grupo en los instrumentos de evaluación.

Asimismo, la Organiza ción de las Naciones Unidas para la Educación, la Ciencia y la Cultura (UNESC O) identific ó las siguientes á reas de interdependencia entre la diversida d cultural y biológica: diversidad lingüística, cultura material, conocimiento y tecnología, modos de subsistencia, relaciones ec onómic as, relaciones sociales, sistema de creencias (UNESCO, 2008). Puede inferirse que esta sáreas de interdepend enc ia representan losvaloresrelacionales de los pueblos originarios y se identific an con su patrimonio biocultural. En este sentido, la teoría de la valoración relacional está estrechamente ligada al patrimonio biocultural de lascomunida des maya-peninsula res.

En este orden de ideas, y con la fina lidad de identific ary configurar indic a dores que contribuyan a la revaloración y fortalecimiento del patrimonio biocultural de las comunidades participantes la metodología que se propone en el presente estudio pemite incorporary 
operacionalizar el a nálisis del impacto de un proyecto sobre las siguientes variables del patrimonio biocultural de la comunidad ejecutora: la diversidad lingüística, las prácticas tra diciona les, los sa beres loc a les, la cosmovisión y la conexión con el lugary las personas.

\section{Materiales y métodos}

El trabajo de campo se realizó desde un enfoque cualitativo, a través de métodos etnográficos (Evers, 2016), con la finalidad de conocer la incidencia de los proyectos relacionadoscon la meliponic ultura en elementos del patrimonio biocultural local. La recolección y a ná lisis de datos se abordó desde un enfoque conceptual del patrimonio biocultural maya peninsular, orientado por las siguientes preguntas:

- ¿Dónde se evidencia el patrimonio biocultural entre los grupos identificados?

- ¿Qué estrategias del proyecto fortalec en (o lesionan) la revaloración del patrimonio biocultural?

- ¿Cómo ha impactado dicha revaloración (o la falta de valoración) la relación entre los grupos partic ipantes y su teritorio?

\section{Recolección de datos para la elaboración de indicadores}

Los datos se recolectaron, mediante un muestreo no probabilístico e intencional, a través de entrevistas grupales e ind ividuales semiestructuradas, observaciones de campo y revisión bibliográfica sobre ca ractenístic as de lossistemastra diciona les de manejo de la región. Se rea lizaron entrevistas con grupos e individuos a sociados con ambas organizaciones: la Escuela Agroecológica de U Yits Ka'an (UYK), en Maní, Yucatán, y Educampo, en Calkiní, Campeche.

La selección de UYK se debió al conocimiento previo que se tenía sobre el trabajo que rea liza la OSC con los grupos de meliponicultores, abordado desde un de enfoque de va loración de la espinitualidad Maya (UYK, $s / f)$. Asimismo, se seleccionaron los grupos a filiados con Educampo, debido a que la región de Los Petenes, donde se encuentran los grupos, constituye la principalá rea donde se conserva esta a ctividad tradic ional de la meliponic ultura en el esta do de Campeche (Pat, Anguebes Franceschi, Pat Femández, Hemández, 2018). El a nálisis de los datos se realizó a través de la clasificación de patrones identificados en memos, observa c iones en campo y segmentos de entrevista, que permitieron la sa tura ción teórica de forma inductiva, según la teońa fundamentada (Chamaz, 2006). Lascategonas emergentes se validaron, a través de grupos focales y fuentes teóricas.

\section{Trabajo de campo}

A través de téc nic as etnográficas a plica das en 2019 se registró la revaloración de componentes del patrimonio biocultural entre grupos de meliponic ultores vinculados con UYK y Educampo. 
Se lleva ron a cabo un total de trece entrevistas semiestruc tura das. Se rea liza ron oc ho entrevista s entre informantes a so cia dosc on UYK, en la s comunidades de Ma ní y Mama, del estado de Yucatán y cinco entre grupos de mujeres organizadas en las comunidades de la región de La Reserva de la Biósfera LosPetenesen Campeche. Asimismo, se llevaron a cabo dos grupos focales, uno en Maní, con un grupo de informantes afiliados a UYK, y otro en Pucnachen con un grupo de informantes afiliadas a Educampo.

Cabe notar, con la excepción de uno, los meliponicultores que partic iparon en el presente trabajo de ambas OSC, representan grupos o gremios compuestos por miembros de la familia extendida que contribuyen al cuidado de las abejas Xunancab. El director de UYK, epigrafistas a socia doscon el proyecto, y coord ina dores del proyec to de a mbas OSC, también contribuyeron al trabajo.

Durante el trabajo de campo se realizaron visitas a cada uno de los meliponarios (colmena res de a bejas nativas tra dic iona les) de los informantes donde llevan a cabo sus a ctividades rela cionadas con la a pic ultura. A tra vés de la observa ción durante las visitas, se abordaron preguntas sobre la percepción de los grupos en relación con sus actividades.

\section{Criterios emergentes de la dimensión biocultural entre grupos de meliponic ultores}

Los datos recolectados evidenciaron una revaloración de los siguientes criterios, constituyentes del patrimonio biocultural maya peninsular, aunado a una valoración mercantilista de la meliponicultura.

\section{Fomento de la lengua Maya}

Durante las entrevistasse observó el fomento de la lengua maya, en diversos contextos. Por ejemplo, a raíz de su participación en los proyectos, los informantes pueden identificar especies de flora y fauna relacionadas con el entomo natural de las abejas Xunancab, como otras especies de abejas nativas, a pesar de que antes no las conocían, así como, otros insec tos que pueden representara menazas para lascolmenas ta mbién se identifican a través de la lengua maya. Asimismo, se identifican otros elementos relacionados con el há bitat de lasabejas, como losholtunes-piedrascónc avas donde beben a gua lasabejas, y el kankab, la tierra utiliza da para sellar los jobones. Asimismo, en lasceremonia srelacionadascon la abeja Xunancab, como el hanlicol-destinada a agradecerpor la cosecha-así como los elementos utiliza dos, como el sacaab, la bebida de maízendulza da con miel y el baalché, una bebida ceremonial, se nombran en lengua maya. Algunos de los nombres dadosa los grupos entrevistados también reflejan el uso de la lengua maya.

\section{La valoración de elementos relacionados c on la cosmovisión}

La promoción de elementos relacionados con la cosmovisión maya peninsular puede evidencia rse en el trabajo de los epigrafistas, quienes buscan interpreta ry soc ia liza r entre grupos de meliponic ultores de UYK la relación de las abejas Xunancab con lo sagrado en la cosmovisión de los antiguos maya-peninsulares. 
Esta revaloración ta mbién se manifiesta en la percepción y en la apropiación de creencias y saberes de los informantes, quienes dicen que las abejas no pertenecen a los meliponicultores, ya que tienen voluntad propia. Es decir, ellas pueden abandonar la colmena cuando quieran, por eso es necesario cuidarlas. Por ejemplo, una creencia común según, informantes meliponic ultores es si un meliponic ultor fallece, esto puede significar el abandono de suscolmenas, a no serque se lesavise a lasabejasque otra persona se hará cargo de ellas. Asimismo, se registró la revalora ción de ceremonias y eventos rela ciona dos con la meliponic ultura. Por ejemplo, meliponic ultores sustentan haber patroc inado o participado en ceremonias de agradecimiento a las abejas Xunancab. En UYK también se está realizando el Día de la Xunancab, en homenaje a la abeja nativa.

\section{Fortalecimiento de prácticas tradicionales y de la comunalidad}

Los hallazgos sugieren que los modos de transferencia de saberes han fortalecido lazos familiares en la comunidad y entre redes de meliponic ultores. Este fortalecimiento se evidencia en la dinámic a de la transferencia de generación a generación, entre miembros de la familia. Asimismo, algunos grupos adoptaron la práctica del «pase en cadena» de las colmenas, forta leciendo los lazos de confianza y de reciprocidad entre los grupos. Además, la participación en movimientos de justic ia a mbiental con el propósito de defender el temitorio y conservarel hábitat de la abeja nativa revela un sentido colectivo de identidad en relación con el temitorio.

Auna do a lo anterior, puede evidenciarse el papel central de la mujer en el manejo y en la toma de decisiones sobre la abeja Xunancab. Por ejemplo, la mayońa de los gruposentrevista dosestán compuestospor mujeresquienes se ponen de acuerdo en lasta reas. Se registró un acontecimiento partic ular en la comunidad de Mama: el médico de la comunidad comenzó a recetar la miel de Xunancab, para aliviar dolores de garganta, conjuntivitis, entre otrasdolencias, favoreciendo el uso de un produc to artesanal y local.

\section{La valoración de los saberes locales}

Los informantes confiman el efec to positivo de la diversific ación de las unidades productivas tales como el huerto, la milpa y el monte, en relación con el cultivo de las abejas nativas, ratific ando así la tradición de uso múltiple de los SSE.

Asimismo, se constató el rescate de saberes relacionados con la vida de las abejas. En Los Petenes, porejemplo, a lgunos informa ntesseña lan que LosPetenes_ec osistemascompuestos por lunares, o islas, de selva intacta-es un gran reservorio de abejas nativas de donde antiguamente se colectaban para llevary criar en los traspatios.

\section{La valoración mercantilista}

Se registró, a demás, entre a lgunos grupos de meliponic ultores una tendencia a enfatiza r la valora ción mercantilista o comercial de la actividad. Dicha valoración puede evidenciarse en los motivos para produc ir miel, por ejemplo, a lgunos de los grupos preferían no consumir la miel sino destinarla a la producción de cosméticos. 


\section{Resultados}

Los criterios emergentes reflejan elementos del patrimonio biocultural relacionadoscon los proyectos de meliponicultura en la península de Yuc atán. Consec uentemente, como estudio de caso se elaboró un instrumento en el que se incomoraron estos criterios para conocerla incidencia de los proyectos sobre los mismos.

Desde una perspectiva del análisis del discurso, los miembros de un grupo nec esitan y utilizan el lenguaje, el texto, la conversación y la comunicación (incluidos aquí en el témino genérico de «disc urso») para a prender, adquinir, modific ar, confimar, artic ular, y también para transmitir persuasivamente las id eologías a otros miembros del grupo, inculcarlas en novicios, y distinguirlas de otras ideologías ajenas a las del grupo (van Dijk, 2000, p. 19). Desde este enfoque, se puede inferirque la meliponic ultura promueve la revaloración de la lengua maya fortaleciendo, a la vez, el sentido de identidad relacionado con el entomo natural y el teritorio.

La cosmovisión incluye «el conjunto de experiencias, conocimientos, mitos, saberes y creencias que dan sustento explic ativo y justific ación a la vida comunal, a las actividades productivasy a los diferentes comporta mientos individua les y colec tivoss (Rendón, 2003, p. 29). Por lo tanto, la reivindic ación de saberes y creencias locales relacionados con la meliponicultura justifican la actividad y las dinámicas sociales y la defensa del temitorio, promoviendo una lógica ecologista con identidad cultural y la comunalidad entre los grupos de meliponicultores y otros habitantes del temitorio.

Para Maldonado (2003), la comunalidad indic a la voluntad individual de ser colectividad y se distingue por la reiteración cíclica, cotidiana y obligatoria de esta voluntad por medio de la participación en las actividades de poder, trabajo, fiesta y relación con el teritorio. Esta organización está fimemente cimentada en un denso tejido social comunitario y regional, constituido por las relaciones festivas y a lianzas de parentesco consanguíneo y ritual, así como por el intercambio recíproco de bienes (p. 7).

Para Ostrom, Ahn y Olivares (2003) dicho modo de vida comunal prioriza el buen manejo de los bienes comunes, a través de la acción colectiva. En este sentido, la interacción por medio de la meliponic ultura, entre los miembros de cada grupo, las comunidades, y las redes sociales propician la confianza y reciprocidad, elementos claves para una buena gobemanza de los SSE.

Auna do a lo anterior, los resultados del trabajo de campo evidencian que las mujeres juegan un papel importante en la meliponic ultura. Según Sobral, La Torre-Cuadros, Alves y Albuquerque (2017) su involuc ramiento direc to en los SSE debe ser promovido, disminuyendo la brecha de lossaberesentre hombresy mujeres; ya que ellasc umplen un pa pel central en la conservación de la biodiversidad (Sobral et al., 2017). 
Desde la óptica de la teoría de la valoración relacional, estas categonáa representan una relación entre el ser humano y la natura leza, a tra vés de valores como: la comunalidad, la identidad, el parentesco, el cuidado y la conexión con la naturaleza (Kleespies y Dierkes, 2020; Schröter et al., 2020). El patrimonio material, por ejemplo, prácticas como la caza, pesca, meliponic ultura, etc.; y el intangible, como son los mitos, leyendas y prácticas religiosas asocia das con especies y ecosistema sespecífic os, ayudan a mantener significados culturales y un sentido de identidad. Entre los grupos de meliponic ultores esto se puede observar en las creencias a socia das con la meliponic ultura y con la defensa del teritorio. Para Daniel et al. (2012), a mbas maneras de relacionarse con la naturaleza - a través de a spec tos materia lese intangibles_a portan valores que contribuyen a su buena administración, a tra vés de activida desy experienc ia sespintua les, estétic asy rec reativas, a prendizaje y de inspiración, así como para revalorary preservar los saberes de los pueblos originariosy locales.

Cabe señalar que todas las dimensiones identific adas contribuyen a la conexión con el lugar y las personas. La conexión con las personas está relacionada con la gestión de recursos no solo por razones de acción colectiva, sino también porque muchas relaciones se basan en la circulación de recursos naturales (por ejemplo, a limentos, materiales de siembra, tierra) dentro de y entre genera ciones, familias y comunida des. Asimismo, los informantes destacaron que las conexiones con las personas pueden no siempre referirse a los seres vivos, pero también a antepasados, inc luida s las ma nifestac iones de a ntepa sa dos en la vida y componentes no vivos de losecosistemas. La conectividad al lugarpuede fomentar el cuidado del temitorio si las personas comparten valores comunes de un lugar (Dacks et al., 2019, p. 7).

\section{La dimensión biocultural en la evaluación de proyectos de meliponicultura}

A partir de las categońas identificadas, se elaboró una matriz como propuesta para incorporar la dimensión biocultural en la evaluación cualitativa del impacto del proyecto, tal como se observa en la tabla 1.

Para la construcción de las categońa s establecidas se sugiere el enfoque cualita tivo esbozado en el a parta do de métodos del presente trabajo. La recolec ción de losdatosse debe realizar a través de técnicas etnográfic as, como entrevistas semiestructuradas, memos y observacionesen campo, comoborando la información reca ba da a travésde gruposfocales. Cabe subrayar que el a nálisis de los datos para deteminar los criterios e indicadores se puede efectuar a través de la teoría fundamentada (Charmaz, 2006); relacionando los criterios e indicadores identific ados a través de la codificación, con las correspondientes va riables.

La figura 1 muestra la interrelación de los indic adores, criterios y va riables con la dimensión bioc ultural. Las c inco va ria bles identific a das, son: diversidad lingüística, prácticas tra dic ionales, saberes locales, cosmovisión, conexión con el lugar y las personas. Mientras que las 
primeras cuatro están relacionadas con el patrimonio biocultural, se incorporó la quinta, que se a poya en la teońa de los valores rela cionales (ver tabla 1). Estas varia bles representan los conceptos centra les del marco sobre el cual se elaboró la propuesta.

Los c riterios se esta blec ieron a partir de las categońa resultantes del a ná lisis del trabajo de campo. Estos se insertan dentro de las variables y reflejan el conjunto de actividades que componen la dimensión biocultural.

Asimismo, los indic a dores-inserta dos en los c riterios-se esta blecieron, a partir del a ná lisis del trabajo de campo. Los ind ic adores reflejan los parámetros específic os que establecen la incidencia del proyecto en el patrimonio biocultural de los grupos.

Cabe hacer hincapié en que este proceso refleja una propuesta que puede ser replicada por los gruposque la adopten con miras a deteminar el impacto de un proyecto sobre la dimensión biocultural.

Figura 1:

Inserción de los indicadores, criterios y variables en la dimensión biocultural.

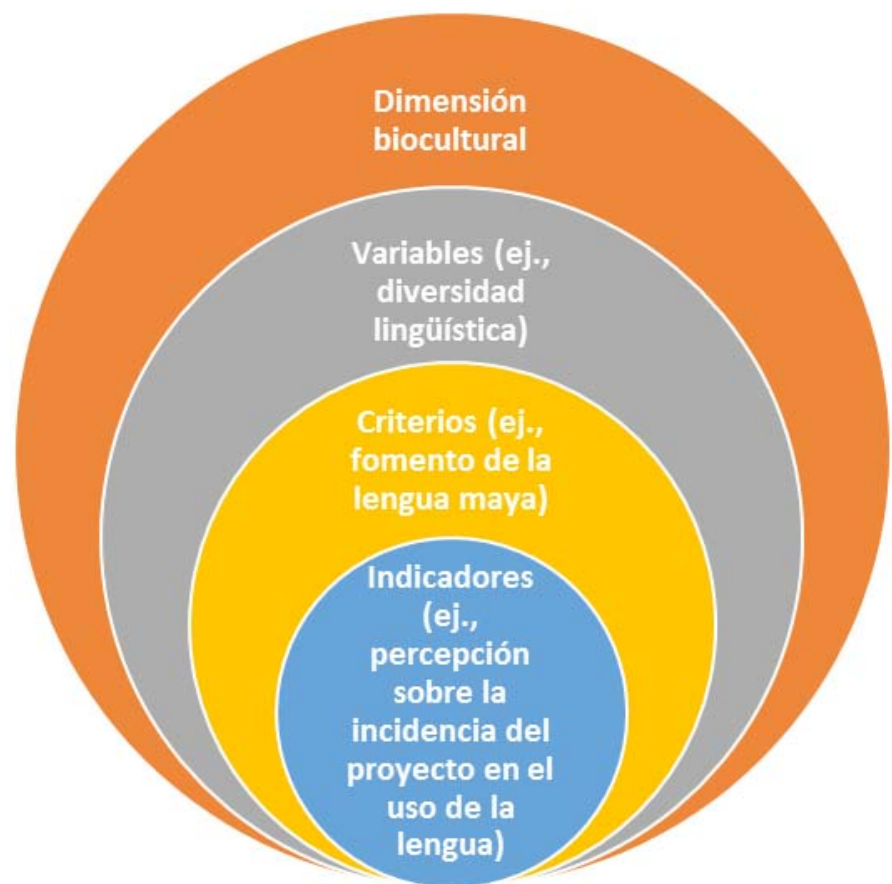

Fuente: Elaboración propia. 
Tabla 1:

Matriz de variables, criterios e indicadores de la dimensión biocultural.

\begin{tabular}{|c|c|c|c|c|}
\hline Variables & Criterios & Indicadores & Preguntas orientadoras & $\begin{array}{c}\text { Técnicas y } \\
\text { fuente }\end{array}$ \\
\hline $\begin{array}{l}\text { Diversidad } \\
\text { lingüística }\end{array}$ & $\begin{array}{l}\text { Fomento de la } \\
\text { lengua maya }\end{array}$ & $\begin{array}{l}\text { Percepción sobre la incidencia del } \\
\text { proyecto en el uso de la lengua maya } \\
\text { en: } \\
\text { intercambio en talleres (grupos) o entre } \\
\text { familia, } \\
\text { flora y fauna; } \\
\text { ceremoniales y } \\
\text { espintuales, medicinales; } \\
\text { nombre de grupos; } \\
\text { otros. }\end{array}$ & $\begin{array}{l}\text { ¿Cómo incide el proyecto de la } \\
\text { meliponicultura en el uso de la } \\
\text { lengua Maya, en todo lo relacionado } \\
\text { con la actividad? }\end{array}$ & \multirow[t]{5}{*}{$\begin{array}{l}\text { Entrevistas semi- } \\
\text { estructuradas a } \\
\text { coordinadores, } \\
\text { y beneficiarios } \\
\text { observaciones } \\
\text { participantes, y } \\
\text { grupos focales }\end{array}$} \\
\hline $\begin{array}{c}\text { Prácticas } \\
\text { tradicionales }\end{array}$ & $\begin{array}{l}\text { Fortalecimiento de } \\
\text { prácticas } \\
\text { tradicionales y de la } \\
\text { comunalidad }\end{array}$ & $\begin{array}{l}\text { Percepción sobre la incidencia del } \\
\text { proyecto en: } \\
\text { La apropiación del uso de objetos } \\
\text { relacionados con prácticas } \\
\text { tradicionales (ej., jobones, meliponario, } \\
\text { etc.); } \\
\text { la participación de familiares; } \\
\text { la participación en las tomas de } \\
\text { decisiones; } \\
\text { la participación de mujeres; } \\
\text { innovaciones culturalmente } \\
\text { apropiadas. }\end{array}$ & $\begin{array}{l}\text { ¿Cómo incide el proyecto en la } \\
\text { apropiación de prácticas } \\
\text { tradicionales y en innovaciones } \\
\text { culturalmente apropiadas, } \\
\text { relacionadas con la meliponicultura, } \\
\text { y en la comunalidad? } \\
\text { ¿Cuáles la motivación principal para } \\
\text { realizar la meliponicultura? (ej., } \\
\text { consumo familiar, comunal, venta, } \\
\text { ceremonial, medicinal) } \\
\text { ¿Cómo se toman las decisiones entre } \\
\text { el grupo? } \\
\text { ¿Tiempo dedicado a la } \\
\text { meliponicultura como actividad } \\
\text { productiva en comparación con } \\
\text { antes del proyecto? } \\
\text { ¿Cómo se contemplan otros sistemas } \\
\text { productivos tradicionales dentro del } \\
\text { proyecto? (lógica de uso múltiple) }\end{array}$ & \\
\hline $\begin{array}{l}\text { Saberes } \\
\text { locales }\end{array}$ & $\begin{array}{l}\text { La valoración de los } \\
\text { saberes locales }\end{array}$ & $\begin{array}{l}\text { Percepción sobre la incidencia del } \\
\text { proyecto en: } \\
\text { la apropiación de saberes } \\
\text { tradicionales; } \\
\text { el intercambio de saberes y creencias } \\
\text { (ej. bioindicadores, medicinales, } \\
\text { ceremoniales); } \\
\text { la transmisión de saberes intra e } \\
\text { intergeneracional; } \\
\text { incorporación de nuevos saberes }\end{array}$ & $\begin{array}{l}\text { ¿Cómo se están revalorizando los } \\
\text { saberes locales relacionados con la } \\
\text { meliponicultura? } \\
\text { ¿El proyecto ha propiciado nuevos } \\
\text { saberes sobre la gestión ambiental? } \\
\text { ¿cuáles son las propiedades } \\
\text { curativas de la miel? } \\
\text { ¿se han hecho cambios en las } \\
\text { actividades productivas, a raíz del } \\
\text { proyecto? (ej. siembra de flora } \\
\text { melifera) }\end{array}$ & \\
\hline Cosmovisión & $\begin{array}{l}\text { La valoración de } \\
\text { elementos } \\
\text { relacionados con la } \\
\text { cosmovisión }\end{array}$ & $\begin{array}{l}\text { Percepción sobre la incidencia del } \\
\text { proyecto en: } \\
\text { la apropiación de mitos y otras } \\
\text { creencias tradicionales; } \\
\text { ceremonias y rituales. }\end{array}$ & $\begin{array}{l}\text { ¿Cómo incide el proyecto en las } \\
\text { creencias relacionadas con la } \\
\text { meliponicultura? } \\
\text { ¿Se ha modificado el significado de } \\
\text { la abeja Xunancab? ¿Cómo? }\end{array}$ & \\
\hline $\begin{array}{l}\text { Conexión } \\
\text { con el lugar } \\
\text { y las } \\
\text { personas }\end{array}$ & $\begin{array}{l}\text { Identidad y valores } \\
\text { compartidos con } \\
\text { personas sobre el } \\
\text { cuidado del } \\
\text { territorio }\end{array}$ & $\begin{array}{l}\text { Percepción sobre la incidencia del } \\
\text { proyecto en: } \\
\text { el sentido de pertenencia al territorio; } \\
\text { lej.. participación en redes sociales en } \\
\text { defensa del territorio, participación en } \\
\text { actividades para la conservación: } \\
\text { el fortalecimiento de la identidad entre } \\
\text { los grupos. }\end{array}$ & $\begin{array}{l}\text { ¿Qué papel tiene la meliponicultura } \\
\text { en la conservación del territorio? } \\
\text { ¿Considera importante la actividad } \\
\text { para el territorio? ¿Por qué? } \\
\text { En este contexto, ¿Cómo repercute } \\
\text { la revaloración de la meliponicultura } \\
\text { en la identidad de los participantes y } \\
\text { en el cuidado del ternitorio? }\end{array}$ & \\
\hline
\end{tabular}

Fuente: Elaboración propia.

En cuanto a la metodología para la aplicación del instrumento, es importante contar con la percepción sobre la situación a ntes del proyec to (a priori) con la finalidad de realizar un ejercic io sobre la incidencia del proyecto en rela ción con su entomo biocultural e inferir una visión a futuro (a posteriori). Es recomendable implementarel instrumento mediante grupos foca les en que los partic ipantes reflexionan sobre la situación a ntes del proyec to con base a los diferentes criterios establecidos, con el propósito de evaluar su incidencia, a futuro, sobre los mismos criterios. 
Las preguntas orienta doras (ver ta bla 1) ayuda ran al modera dor a a bordar los temas pertinentes a la dimensión biocultural. Asimismo, los grupos focales también permiten a nimar y mantener la dinámic a colectiva. Los resulta dos serán presentados de forma descriptiva y na rra tiva y permitirán esta bleceruna percepción colec tiva de losgrupos, si el proyecto está encaminado hacia la bioculturalidad y visualizar sus posibles impactos a futuro $\mathrm{y}$, de ser necesario, hacer los ajustes que se juzguen convenientes.

Las preguntas orienta doras presentadas en la matriz pueden utiliza rse como cata liza doras de los gruposfocales con la finalidad de:

- dary recibir información colec tiva sobre los indic a dores propuestos,

- debatir temas de importancia para la comunidad relacionadoscon los indicadores,

- recibir la conformidad del grupo para la aplicación del instrumento,

- validar los resulta dos de las evalua ciones y

- formular recomendaciones.

Por tanto, el instrumento propuesto tendrá por objeto ponera disposición de los toma dores de decisiones una evaluac ión de juic io colec tivo de la situa ción previa al proyecto, a partir de la percepción de losgruposbeneficiariossobre sus efec tos y su incidencia en los diferentes componentes de la dimensión biocultural.

\section{Disc usión}

La falta de métodos de evaluación que incorporen elementos del patrimonio biocultural en proyectos que afectan directa o indirectamente la buena gestión y conservación de la diversidad bioc ultural es una ca rencia que debe ser colmada. Esto es importa nte si se considera que los métodos de evaluación convencionales tienen un sesgo mercantilista o, en el mejor de los casos incomoran componentes ambientales, pero, en general, dejan por fuera los impactos, positivos y negativos, que el proyecto puede tener sobre el patrimonio biocultural de las comunidades involucradas.

El enfoque de la teoría de la valoración relacional contribuye a fortalecer la conexión y la relación entre las personas, entre las personas y el teritorio y su patrimonio biocultural. El instrumento propuesto, por tanto, busc a contribuir al fortalecimiento y conservación de la dimensión bioc ultural en proyectos de gestión a mbiental, particularmente en regiones que cuentan con altos índices de diversidad biocultural, como la península de Yucatán.

Por su enfoque multidisciplina rio y empírico, el instrumento propuesto no pemite su réplica en otros contextos sin antes elaborar un diagnóstico de la problemática del proyecto particular a evaluar, desglosando con el mayor detalle posible los componentes de tipo biocultural que entrarán en juego en el proyecto. Esto se debe a que los indicadores bioc ultura les están necesa ria mente vincula doscon el luga ry el c ontexto particular del pro- 
yec to considerado. No obstante, la literatura sobre la consenvación biocultural puede proveer guías de onienta ción para elaborar los indic adores (DeRoy et al., 2019).

Se recomienda, por lo tanto, una selección de indic adores emergentes ad hoc, según el caso partic ulara evaluar. Auna do a la exigencia transdisc iplinaria que implica el diseño del instrumento para este tipo de proyectos, se recomienda una estrecha participación en los gruposfocales, en todas las etapas de la evaluación. Cabe acla rarque la racionalidad de estas guías no depende de la escala o dimensión del proyecto, es decir, son a plicables a mic ro y mega proyectosy están orienta dasa loscomponentes relaciona doscon el impac to en el patrimonio biocultural en el área del proyecto particular.

El instrumento se aborda desde la percepción de los grupos, ya que esta depende fundamenta Imente del conoc imiento cultural y de la experiencia local, los cuales representan la manera en la que lascomunidadeslocales perc iben los efectos de las interacciones humanas con la natura leza en un contexto ecológic o (Dacks et al., 2019, p. 7).

Adic ionalmente, los indicadores de percepción permiten evaluar la manera en la que los individuos o la comunidad reaccionan ante asuntos de política pública, gestión o gobemanza relacionados con cambios de estado ambiental o ecológicos en contextos cambiantesy de alta vulnera bilidad. En este sentido, eva lua rlaspercepciones loca les puede ser un factor que, a pesar de ser signific ativo, suele ser omitido en los marcos de evaluación de gestión ambiental. Asimismo, debido a que los indica dores de percepción son subjetivos, estos reflejan de una manera intrínseca la percepción del bienestar (Dacks et al., 2019, p. 7).

El instrumento de evaluación propuesto para el caso de la meliponicultura no contempla su valoración monetaria, en apoyo a la desmercantilización de este tipo de actividades, cuya esencia yace en una racionalidad biocultural, mucho más amplia, compleja y heterogénea que la sola racionalidad mercantilista. Lo anterior se debe a que la tendencia a la comodificación de la naturaleza está rela cionada con la lógica neoliberal y su incidencia en la apropiación merc a ntilista de los rec ursos natura les (Durand, 2014). En este sentido, desde una óptica de la ecología política, el encuentro de las dos racionalidades_la mercantilista y la biocultural-ha propiciado un conflicto porel rescate de la meliponicultura. Se considera, finalmente, que la aplic ación participativa del instrumento permitirá planificarlasactividadesdel proyec to de forma culturalmente apropiada, teniend o presente que los pueblos origina rios tienen el derec ho ina lienable a determinar sus priorida des y elaborar estra tegias para la consecución de su bienestar (ONU, 2007).

\section{Agradecimientos}

UNAM, Becario(a) del Programa de Becas Posdoctorales en la UNAM, Centro Peninsular de Huma nida des y Ciencias So cia les (Cephc is-UNAM) / Dr. Miguel Ángel Pinkus Rendón. 


\section{Referencias}

Allen, K.E., Quinn, C.E., English, C. y Quinn, J.E. (2018). Relational values in a groecosystem govemance. Curr. Opin. Environ. Sustain. 35, 108-115. doi: 10.1016/j.c o sust.2018.10.026.

Sobral, A., La Torre-Cua dros, M., Alves, R, y Albuquerque, U.P. (2017). Conservation efforts based on local ecological knowledge: The role of social variables in identifying environmental indicators. Ecological Indicators. 81, 171-181. doi: 10.1016/j.ec olind.2017.05.065

Bridgewater, P. y Rotherham, I. (2019). A critical perspective on the concept of biocultural diversity and its emerging role in nature and heritage conservation. People and Nature. 1:291-304. doi: 10.1002/pan3.10040.

Chan, K. M. A., Balvanera, P., Benessaiah, K., Chapman, M., Díaz, S., GómezBaggethun, E., et al. (2016). Why protect nature? Rethinking values and the environment. Proc. Natl. Acad. Sci. 113, 1462-1465. doi: 10.1073/ pnas. 1525002113.

Chamaz, K. (2006). Constructing Grounded Theory: A Practical Guide Through Qualitative Analysis. London, UK: SAGE.

Chá vez, M. (2014). El huerto familiar maya. En A. Sánchez Suárez y A. García Quintanilla (eds.), La casa de los mayas de la península de Yucatán: historias de la maya naj, (pp. 257-293). Mérida. México: UADY.

Comisión Nacional de Areas Naturales Protegidas, CONANP (s.f.) Estrategia Nacional para un Desarrollo Sustentable del Turismo y la Recreación en Áreas Proteg idas de Méxic o, 2007-2012, Comisión Nacional de Áreas Natura les Protegidas-Secretańa de Medio Ambiente y Recursos Naturales, México D. F.

Consejo Nacional de Evaluación de la Política de Desarrollo Social, CONEVAL (s.f.). Evaluación de la política social: ley general de desarrollo social. Recuperado de: https://www.coneval.org.mx/Evaluacion/NME/Paginas/ LeyGeneral.aspx

Da niel, T.C., Muhar, A., Amberger, A., Aznar, O., Boyd, J .W., Chan, K.M.A., Costanza, R., Elmq vist, T., Flint, C.G., G obster, P.H. et al. (2012). Contributions of c ultural services to the ecosystem services agenda. Proc Nat Acad. Sci. 109:88128819. doi: 10.1073/pnas.1114773109.

DeRoy, B., Da rimont, C., y Service, C. (2019). Biocultural ind ic a tors to support locally led environmental management and monitoring. Ecology and Society 24(4):21. doi: 10.5751/ES-11120-240421.

Dacks, R., Tic ktin, T., Mawyer, A., Caillon, S., Claudet, J., Fabre, P., J upiter, S.D., McCarter, J., Mejia, M., Pasc ua, P., Sterling, E., Wongbusa ra kum, S., (2019). Developing biocultural indicators for resource management. Conservation Science and Practice; e38:11. doi: 10.1111/csp2.38.

Durand, L. (2014). ¿Todos ganan? Neoliberalismo, naturaleza y consenvación en Méxic o. Sociológica. 29(82), 183-223. Recupera do de: http:// ww w.scielo.org.mx/ scielo.php? script =sci_a rttext\&pid =S0187$01732014000200006 \&$ Ing =es\&nm=iso \&tlng =es

Figueroa, F., Guzmán, M. G. (2011). La ecología política en México. ¿Dónde estamos y para dónde vamos? Estudios Sociales, 19(37), 282-307. Recupera do de: http:// www.scielo.org.mx/ scielo.php? sc ript=sci_arttext\&pid= S0188-45572011000100011\&lng =es\&nm=iso \&tlng =es

Escobar, A. (2015). Temitorios de diferencia: la ontología política de los «derechos al territorio». Desenvolvimento e Meio Ambiente 35. doi: 10.5380/ dma.v35i0.43541.

Evers, J. (2016). Ela borating on Thick Analysis: About Thoroughness and Creativity in Qualitative Analysis. FQS 17(1), Art. 6. doi: http://dx.doi.org/10.17169/fqs17.1.2369.

Galván-Miyoshi, Y. (2008). Integración de indicadores en la evaluación de sustentabilidad: de los índices agregados a la representación multic riterio (pp.95-118). En M. Astier, O.R. Masera y Y. Galván-Miyoshi, Evalua ción de sustentabilidad. Un enfoque dinámic o y multidimensional. México: UNAM.

Masera, O. y López-Ridaura, S. (2008). Las evalua c iones de sustenta bilidad (pp. 41-58). En M. Astier, O.R. Masera y Y. Galván-Miyoshi, Evalua ción de sustentabilidad. Un enfoque dinámic o y multidimensional. México: UNAM. 
García-Barrios, R. y García-Barrios, L. (2008). La sociedad controlable y la sustentabilidad. En M. Astier, O.R. Masera y Y. Galván-Miyoshi, Evalua ción de sustentabilidad. Un enfoque dinámico y multidimensional (pp. 173-192). Ciudad de México, Méxic o: UNAM.

González, J. y de Araujo Freitas, C. (2014). El solar de la casa maya y su diversa a pifauna. En A. Sánchez Suárez y A. García Quinta nilla (eds). La casa de los mayas de la península de Yucatán: historias de la maya naj (pp. 241-255). Mérida, México: UADY.

y Quezada-Euán, J.j. (2010). Producción tra dicional de miel: abejas nativas sin aquijón (trigonas y meliponas). En R. Durán García y M.E. Méndez González (eds). Biodiversidad y Desa rollo Humano en Yucatán (pp. 382-384). Mérida, México: CICY, A.C.

Queza da-Euán, J.J. y Medina-Medina, L. (2006). New perspectives for stingless beekeeping in the Yucatan: results of an integral program to rescue and promote the activity. J oumal of Apicultural Research 45(3), 234-239. doi: 10.3896/IBRA.1.45.4.14.

Hill, R., Nates-Parra, G., Quezada-Euán, J J., Buchori, D. LeBuhn, G., Maués, M. Pert, P., Kwapong, P., Sa eed, S., Breslow, S., et al. (2019). Biocultural approaches to pollinator conservation. Nature Susta inability. 2: 214-222. doi: 10.1038/s41893-019-0244-z.

Hirst, K. (2018). Ancient Maya Beekeeping. ThoughtCo, Recuperado de: http:// www.thoughtco.com/ancient-maya-\%20beekeeping-169364

Intergovemmenta I Sc ience-Polic y Platform on Biodiversity and Ec osystem Services, IPBES (2018). The regional assessment report on biodiversity and ecosystem servic es for Euro pe and Central Asia. Summa ry for polic yma kers. En M. Fisc her, M. Rounsevell, A. Torre-Marin Rando, A. Mader, A. Church, M. Elbakidze, V. Elias, T. Hahn, P.A. Ha mison, J. Ha uck, B. Martín-López, I. Ring, C. Sa nd ström, I. So usa Pinto, P. Visconti, N.E. Zmmemann y M. Christie (eds.). The regional a ssessment report on bio diversity and ecosystem services for Europe and Central Asia, (pp. XV-XXIII) Doi: https://doi.org/10.5281/zenodo.3237428

Kleespies, M.W. y Dierkes, P.W. (2020). Exploring the Construct of Relational Values: An Empinical Approach. Front. Psychol. 11: 209. doi: 10.3389/fpsyg.2020.00209.

Loh, J. y Hamon, D. (2005). A global index of biocultural diversity. Ecological Indic ators 5, 231-241. doi: https:// doi.org/10.1016/j.ecolind.2005.02.005.

López, M. (2015). Resistencias a groecológicas en Yucatán, Méxic o. Ecología Política. Cuademos de debate intemacional. 49: 119-118. Recuperado de: https:// www.ec ologia politic a .info/ $? p=2272$.

Maffi, L. (2014). Biocultural Diversity. The True Web of Life. En L. Maffi y O. Dilts (eds.). Biocultural Diversity Toolkit. Introduction to Biocultural Diversity, vol.1, (pp. 6-16). British Columbia: Terralingua.

Ma in, D. (2012). A Different Kind of Beekeeping Takes Flight. New York Times, 17 de febrero. Recuperado de: https://green.blogs.nytimes.com/2012/02/17/adifferent-kind-of-beekeeping-takes-flight/? mtrref=www.google.com\&g wh= D18EC AE7FB6C ED7C 582BEE962761A 095\&g wt=pa y\&a ssetType =REG IWALL.

Maldonado, B. (2003). Introduc ción: La comunalidad como una perspectiva a ntropológica india. En J.J. Rendón (ed.). La Comunalidad: Modo de vida en los pueblos indios (pp. 5-13). Ciudad de Méxic o, Méxic o: CONACULTA.

Martínez-Alier, J., Munda, G., y O'Neill. (1998). Weak Comparability of Values as a Foundation for Ecological Economics. Ecological Economic S, 26, 277-286. doi: https://doi.org/10.1016/S0921-8009(97)00120-1.

Schröter, M., Ba -ak, E., Christie, M., Church, A., Keune, H., Osipova, E., OterosRozas, E., Sievers-Glotzba ch, S., va n Oudenhoven, A., Ba lva nera, P., González, D., J a cobs, S., Molnár, Z, Pascual, U. y Martín-López, B. (2020). Indic a tors for relational values of nature's contributions to good quality of life: the IPBES approach for Europe and Central Asia. Ecosystems and People 16(1), 50-69. doi: https://doi.org/10.1080/26395916.2019.1703039.

Ochoa, S.M. (2007). La Ley General de Desamollo Social y la medición de la pobreza. Centro de Estudios So ciales y de O pinión Pública. Cámara de Diputados, LX Legislatura. Mexico.

Organización para la Cooperación y el Desarrollo Económico, OECD (2003). Environmental ind ic a tors. Development, mea surement and use. Organization 
for Ec onomic Co-operation and Development. Pańs: OECD. Recuperado de: http:/ / www.oecd.org/ environment/ ind ic a tors-modelling-outlooks/ 24993546.pdf

Organiza ción de Naciones Unidas, ONU (2007). Declaración de las Naciones Unidas sobre los derechos de los pueblos indígenas. Departamento de Asuntos Económic os y Sociales Pueblos Indígenas. Recuperado de: https:// www.un.org/development/desa/ind igenous-peoples-es/decla racion-sobrelos-derechos-de-los-pueblos-indigena s.html

Ostrom, E., Ahn, T., y Olivares, C. (2003). Una perspectiva del capital social desde las ciencias sociales: capital social y acción colectiva. Revista Mexicana de Sociología, 65(1). Recuperado de: http:// www.scielo.org.mx/ sc ielo.php? sc ript=sc i arttext\&pid=S0188-25032003000100005

Otero, I. Fa rrell, K., Pueyo, S., Ka llis, G., Kehoe, L., Haber, H., Plutzar, C., Hobson, P., García Márquez, J., Rodríguez Labajos, B., Martin, J .Let al. (2020). Biodiversity polic y beyond economic growth. Conservation Letters; e12713. doi: https:// doi.org/10.1111/conl.12713

Pat, L., Anguebes Franceschi, F., Pat Femández, J .M., Hemández, P. (2018). Condición y perspectivas de la meliponic ultura en comunidades mayas de la resenva de la biósfera Los Petenes, Campeche, México. Estud. cult. Maya, 52, 227-254. doi: http://dx.doi.org/10.19130/iifl.ecm.2018.52.939.

Pearson, G. (2014). Women Work to Save Native Bees of Mexico. Wired. 5 de marzo. Rec upera do de: https://www.wired.com/2014/03/women-work-savena tive-bees-mexic o/

Quezada-Euán, J.J. (2018). The Past, Present, and Future of Meliponiculture in Mexico. En J.J. Queza da-Euán (ed.). Stingless Bees of Mexico. The Biology, Management and Conservation of an Ancient Heritage, (pp. 243-269). Springer Intemational Publishing.

Ramírez, L.A. (2006). Impacto de la globaliza ción en los mayas yucatecos. Estudios de cultura maya, 27, 73-97. Recuperado de: https://www.redalyc.org/ a rtic ulo.oa id $=281322927004$

Rendón, J.J. (2003). La Comunalidad, Modo de vida en los pueblos indios, Ciudad de México, Méxic o: CONACULTA

Rodríguez-Ba lam, E. y Pinkus-Rendón M. Á. (2015). A picultura, Entomo y Modemidad en Localida des de Yuca tán, Méxic o. Biotemas 28(3):143-157. doi: 10.5007/ 2175-7925.2015v28n3p143.

Rodríguez-Ortega, C., y Flores-Martínez, A. (2018). El Sistema Nacional de Indic a dores Ambientales (SNIA). En J. López Blanco y M. Rodríguez Ga miño (coords.) Desarrollo de indicadores ambientales y de sustentabilidad en México (pp. 15-26).

Rundle, H. (2019). Indigenous Knowledge Can Help Solve the Biodiversity Crisis. Scientific American, 12 de octubre. Recuperado de: https:// blogs.scientific a meric a n.com/observations/ind igenous-knowledge-can-helpso Ive -the - $b$ io d ive rsity-c risis/ ? fb clid =Iw A R IBFIAEFjrUV p Q 5 UZ5 Wyto NWd9meipJ 5ciCAMlv 25zWC nVSSylJ vyC jU

Sa rukhán, J. (2009). Las reservas ecológic as insufic ientes para proteger la biodiversidad nacional. Boletín UNAM, 528, Ciudad de México, México: UNAM. Recuperado de: www.dgcs.unam.mx/boletin/bdboletin/2009 528.html

Stig litz, J . (2019). It's time to retire metric s like GDP. They don't mea sure everything that matters. The Guardian, 24 noviembre del 2019. Recupera do de: https:/ / w w w.the gua rdia n.com/c o mmentisfree/2019/ nov/24/metric s-gdpeconomic-perfomance-social-progress

Toledo, V. y Barrera-Bassols, N. (2008). La memoria biocultural. La importancia ecológica de las sabiduńas tra dic ionales. Barcelona, España: Icania.

Organiza ción de las Naciones Unidas para la Educa ción, la Ciencia y la Cultura, UNESCO (2008). Links between biological and cultural diversity-concepts, methods and experiences. Report of an International Workshop. Paris: UNESCO 47.

U Yits Ka'an, UYK. S.f. ¿Qué hacemos? U Yits Ka'an. Recupera do de: https:// www.uyitskaan.org/que-hacemos/ van Dijk, T. 2000. Ideología. Un enfoque multidisciplinario. Barcelona, España: Gedisa Editorial 